\title{
Nombrar y Clasificar: Aproximación a una Epistemología de las Clases Sociales
}

\author{
NAMING AND CLASSIFYING: AN APPROACH TO THE EPISTEMOLOGY OF SOCIAL CLASSES
}

Dra. Emmanuelle Barozet (ebarozet@uchile.cl) Departamento de Sociología, Universidad de Chile (Santiago, Chile)

Dr. Oscar Mac-Clure (oscar.macclure@gmail.com) Centro de Investigación Sociedad y Políticas, Universidad de Los Lagos (Santiago, Chile)

\begin{abstract}
In this paper, we provide an analysis about the relationship between expert and pragmatic categories used to name and categorize social space, from the point of view of the social hierarchies that structure it. Taking the case of Chile, and applying an experimental simulation methodology, we seek to contribute to the epistemological debate about the representations that individuals elaborate about social space, from a cognitive and interactionist perspective of everyday life. We analyse in particular the scope of the concept of social class as an element of expert description of social reality, but also as a categorization used by ordinary subjects. We argue that although classifications used by ordinary people to describe members of society are not consistent with the most common academic class schemes, they nevertheless share the same logic and ranking system, adding evaluative and normative elements to the cognitive distinction, in the social construction of reality.
\end{abstract}

Key words: epistemology, social class, categorization, social inequality, Chile

\section{Resumen}

En este artículo, presentamos un análisis acerca de la relación entre categorías expertas y categorías pragmáticas usadas para nombrar y categorizar el espacio social, desde el punto de vista de las jerarquías sociales que lo estructuran. Tomando el caso de Chile y aplicando una metodología experimental de simulación, aportamos al debate epistemológico acerca de las representaciones que los sujetos sociales elaboran acerca del espacio social, desde una perspectiva cognitiva e interaccionista situada en la vida cotidiana de las personas. Analizamos en especial el alcance del concepto de clase social en cuanto elemento de descripción experta de la realidad social, contrastándolo con la categorización de la misma de parte de sujetos comunes y corrientes. Postulamos que si bien las clasificaciones que ocupan las personas comunes y corrientes para describir a los integrantes de la sociedad no se condicen con las clasificaciones académicas más comunes, comparten sin embargo las mismas lógicas clasificatorias de jerarquización y ordenamiento, agregándose a la distinción cognitiva elementos normativos que aportan a la construcción social de la realidad.

Palabras clave: epistemología, clase social, categorización, desigualdad social, Chile. 


\section{Introducción}

En el ámbito de la epistemología de las ciencias sociales, entendida como la reflexión acerca de los fundamentos y métodos del conocimiento científico, la pregunta por la representación de los sujetos sociales en el espacio social, en especial el cómo nombrar y clasificarlos, ha sido de gran relevancia. Sin embargo, son escasos los trabajos que analizan el problema epistemológico de la relación entre, por un lado, las categorías expertas que circulan en la academia, los medios y la política pública y, por el otro, las categorías que las personas movilizan desde una perspectiva cognitiva e interaccionista en la vida cotidiana para pensar el espacio social.

En el campo más específico de la sociología, la clase social ha sido un concepto central desde el siglo XIX, por su aporte a la reflexión sobre la estructura social y las desigualdades sociales, tanto en su calidad de fenómeno social como forma de categorización y representación. En efecto, las clases sociales, junto con una serie de términos sinónimos, sea grupo social, capa, grupo socioeconómico o estrato para la época moderna u otros términos como casta y estamento, para períodos pre modernos, son una de las principales representaciones de las cuales disponen las personas para nombrar y describir los grupos sociales cuando piensan la sociedad. Al igual que muchos otros conceptos de uso corriente tanto en ciencias sociales como en el ámbito mismo de la vida, la clase social es una categoría que simplifica la realidad y que usamos para orientarnos y actuar en nuestro entorno. Además de la descripción de los grupos que componen la sociedad, ofrece una representación de las jerarquías que existen dentro de la misma. Como otros, la clase social no es un concepto cerrado y estable, sino que altamente dinámico. Refleja las formas de categorizar la sociedad de parte de los cientistas sociales, siendo también un concepto apropiado, discutido por los integrantes de la sociedad, por los medios y por los actores sociales movilizados: el concepto es discutido, asimilado, rechazado por los mismos individuos, o puesto en comparación y competencia con otras visiones del espacio social.

La reflexión que presentamos a continuación se enmarca en una de las grandes y constantes interrogantes epistemológicas, teóricas y empíricas acerca de la relación entre las categorizaciones sociales como concepto y como objeto real, es decir, si las categorizaciones corresponden a una mera clasificación social abstracta que usan los investigadores en ciencias sociales (nominalismo) o si tienen un correlato en la realidad social como objetos reales (realismo). Nuestro objeto de atención será esto último, la observación de las categorizaciones que surgen de los propios sujetos, que denominaremos categorías "pragmáticas". Partiendo de esta base, las preguntas que se desarrollan en este artículo son las siguientes: desde la epistemología de las ciencias sociales, ¿qué relación existe entre clasificaciones expertas y clasificaciones pragmáticas para nombrar y clasificar a los integrantes de la sociedad? ¿Comparten las mismas lógicas clasificatorias? ¿Son transversales al conjunto de la sociedad o existen distintas representaciones en distintos grupos sociales? ¿Qué ocurre con la clase social, como forma histórica privilegiada de describir la sociedad pero que puede haber perdido fuerza frente a las nuevas formas de estructuración de la sociedad?

Nuestro trabajo se sitúa en el caso de Chile. La sociedad chilena, caracterizada por fuertes jerarquías ancladas en la historia, es escenario de un intenso debate acerca de las desigualdades y las relaciones entre grupos sociales, en un momento en que la terminología propia del marxismo en torno a la noción de clase social, hegemónica hasta los años 1970, parece haber cedido espacio a comprensiones más difusas de las relaciones entre grupos sociales. Realizamos esta reflexión en el marco de la aplicación de un método de investigación de tipo experimental, que genera información sobre las formas en que las personas comunes y corrientes nombran los objetos de la realidad y se figuran la sociedad. Postulamos 
que si bien las clasificaciones que ocupan las personas "comunes y corrientes" para describir a los integrantes de la sociedad no se condicen con las clasificaciones académicas más comunes, comparten sin embargo las mismas lógicas clasificatorias de jerarquización y ordenamiento, pues la categorización realizada se asemeja a una clasificación según clases sociales, agregándose a la distinción cognitiva elementos normativos acerca de la construcción de la sociedad.

Primero, revisaremos el debate en torno a los conceptos que usamos para nombrar y clasificar y al elaborar representaciones de la sociedad, analizándolos en su doble vertiente de categoría y objeto. Luego, sintetizaremos el debate epistemológico sobre la relación entre clase social como categoría y objeto en ciencias sociales, antes de presentar algunas formas de medición de las clases sociales. A continuación, describiremos una metodología de investigación que permite analizar la distancia entre la categoría clase y el objeto clase. Finalmente, presentaremos resultados y reflexiones en torno al ejercicio de nombrar y clasificar a los grupos sociales para el caso de Chile.

\section{Categorizaciones, clasificaciones y la pregunta por la relación entre categorías y objetos en ciencias sociales}

Para las ciencias sociales, la creación de clasificaciones, nomenclaturas, tipologías, esquemas, conceptos y codificaciones responde a la necesidad de disponer de artefactos que mediatizan, categorizan y representan la sociedad (Durkheim y Mauss 1903). La categorización consiste en una actividad cognitiva que agrupa objetos, hechos o eventos no idénticos en categorías (Mervis y Rosch 1981). Las categorías son a su vez consideradas como conjuntos de objetos, hechos o eventos, que las personas consideran equivalentes. El concepto de categorización es central para comprender los procesos de representación social, debido a que se refiere al contenido y a la organización de las categorías (Salès-Wuillemin 2007). Por su lado, Moscovici señala que una "representación está compuesta de figuras y expresiones socializadas" y destaca que "la representación social es una modalidad particular del conocimiento, cuya función es la elaboración de los comportamientos y la comunicación entre los individuos" (Moscovici 1979:8). Las clasificaciones, por su lado, dan cuenta de los actores, sus prácticas y sus posiciones en el espacio social, lo que se logra con más o menos eficiencia y más o menos poder heurístico (Grusky 2001). Al respecto, en la actualidad existe un renovado interés por las representaciones sociales, las fronteras simbólicas entre grupos, así como el arte de nombrar y clasificar, incluso en Chile (Méndez 2008, Joignant y Guell 2009). Para la reflexión acerca de las formas en que nombramos y representamos la realidad social y sus objetos, es crucial entonces tomar en consideración la capacidad de comprensión y transformación del espacio social de los sujetos, debido a su propia reflexividad. Como lo señala Giddens, "todos los actores son teóricos sociales, y todos deben serlo para ser de alguna manera agentes sociales. Las convenciones a las cuales recurrimos en la organización de la vida social nunca son 'hábitos ciegos'. Una de las contribuciones distintivas de la fenomenología [...] es que ha mostrado que 1) la conducta de la vida social implica continuamente una 'teorización' y 2) incluso los hábitos más duraderos, o las normas sociales más inquebrantables, implican una atención reflexiva continua y detallada" (Giddens 1993:5). En esta perspectiva, se considera que las categorías y estructuras sociales no son externas a los actores sociales, buscando de esta manera romper con el dualismo corriente en ciencias sociales entre estructura y agencia.

Nuestro punto de partida dentro de la reflexión epistemológica en torno a la construcción de la relación entre realidad y las percepciones de las personas, es el paralelismo entre visiones expertas y visiones pragmáticas, en un contexto en el cual existen pensamientos e intencionalidad de parte de los sujetos. El debate sobre lo que son y cómo surgen las clasificaciones sociales ha tenido desarrollos muy extensos, 
pero buscamos enmarcar la reflexión con breves menciones a dos de los principales representantes de la reflexión acerca de la construcción de la relación social, Schütz y Giddens. En los años 1930, Schütz señala que la epistemología tiene como una de sus preocupaciones la relación entre analista social y actor social en la medida en que la reflexividad de las personas juega un papel central en la construcción de la vida social. Como lo señala la psicología social y cognitiva, tanto los actores sociales como los analistas construyen mentalmente modelos de representación de la sociedad, en base a informaciones más o menos parciales que circulan entre individuos y en espacios colectivos, y que por lo tanto no pueden dar cuenta de la totalidad de lo social. En este sentido, los conceptos que usan las personas son "estructuras cognitivas que consisten en un conjunto de componentes inscritos en las relaciones entre las personas" (Coxon y Jones 1979:124), cuya complejidad puede variar ampliamente según los contextos, las personas y los grupos sociales a los cuales pertenecen.

Nos referimos igualmente a la reflexión de Giddens sobre la doble hermenéutica de las ciencias sociales y la mediación que significa el lenguaje, particularmente en la elaboración de categorías. Como es sabido, este enfoque releva los efectos mutuos entre el sujeto y la realidad social en sus múltiples relaciones: "Esto es la expresión lógica de la doble hermenéutica: los actores comunes son seres productores de conceptos y esos conceptos son constitutivos de lo que hacen. Los conceptos de la ciencia social no pueden quedar aislados de su potencial aprobación e incorporación en la acción cotidiana" (Giddens 1993:13). Es decir, a diferencia de las ciencias básicas, las ciencias sociales se enfrentan a la complejidad de objetos de estudio que tienen su propia reflexividad, lo que implica una dinámica intersubjetiva. Una parte importante de la epistemología busca entender además el paso de concepciones individuales o aisladas a "lo social" como un todo, un continuo. Existe por lo tanto una complejidad adicional en la investigación social, pues aunque reflexivo, el individuo nunca se puede salir del flujo de la acción. En breve, "nuestras disciplinas presuponen dos elementos: la actividad social humana y la intersubjetividad. Los conceptos que usamos se forjan por lo tanto en el ir y venir entre expertos descifrando la realidad social y el mundo que estudian. No se trata de un mundo dado y fijo, sino que un mundo que se forja constantemente y que responde a las categorías con los cuales los expertos lo describen" (Giddens 1993:8).

En una línea más reciente de esta reflexión, Boltanski (2009) sostiene que la corriente principal de la teoría crítica parte del supuesto de un desconocimiento de los propios actores acerca de las condiciones sociales que experimentan, siguiendo las teorías de la alienación de Marx y de sus seguidores. En ruptura con Bourdieu, Boltanski propone un giro epistemológico hacia una sociología "pragmática", adoptando el punto de vista de los actores y sus propias visiones de la sociedad. Plantea que el mundo de su experiencia puede llegar a ser contradictorio con las clasificaciones expertas formuladas por las instituciones establecidas. En un espacio más cercano desde el punto de vista geográfico, Boaventura de Sousa Santos plantea la necesidad de construir una "epistemología del sur", que incluye alterar desde la práctica de quienes ocupan posiciones subalternas, las lógicas establecidas de clasificación social que desde el poder institucionalizado naturalizan desigualdades y jerarquías sociales. En general, un enfoque de este tipo busca insistir en la capacidad de los sujetos para transformar el espacio social utilizando o activando las categorías de las cuales disponen.

Para este artículo, nos insertamos específicamente en la reflexión siguiente: el modo en que los actores hacen referencia a entidades colectivas en la sociedad, constituye una forma de cualificarla y al hacerlo, contribuyen a construirla (Boltanski y Thévenot 1991, Hacking 1999). En este marco, aplicando la distinción de Swidler y Arditi (1994) entre el conocimiento formal y el desarrollado por las personas en su vida cotidiana, frente al conocimiento dotado de la autoridad de las instituciones acerca de las 
divisiones sociales, nuestra interrogante se refiere a si ese conocimiento tiene un correlato en el conocimiento usado por las personas en sus prácticas cotidianas para diferenciar a las personas en la sociedad. Esto conduce a precisar la problemática inicial y preguntarnos específicamente en qué medida el conocimiento usado por las personas para distinguir categorías sociales en la sociedad chilena, coincide o no con las categorizaciones acerca de las diferencias sociales en general. Es decir, si tienen un correlato en las subjetividades de las propias personas o bien si entre éstas predomina una lógica práctica que distingue de otro modo a las personas en la sociedad. Exige observar de modo pragmático las generalizaciones utilizadas habitualmente en las relaciones interpersonales, las denominaciones más populares, el sentido común aplicado habitual o cotidianamente al categorizar, sea en altos o bajos niveles de reflexividad (Boltanski 2009).

Para ello, establecemos una diferencia entre clasificaciones expertas y clasificaciones pragmáticas. La expresión "categorías objetivas" otorga un cariz omnisciente y normativo al canon establecido por taxonomías académicas, tipologías científicas, económicas, sociológicas e incluso estudios de mercado, por lo que en su lugar utilizaremos el término más neutral de clasificaciones "expertas" (Brousse et al. 2010). En contraste, denominamos como clasificaciones "pragmáticas" a las categorías que surgen de los propios sujetos y las taxonomías que usan las personas en la vida cotidiana como referentes para describir la sociedad, sea desde cada individuo en particular o en la interacción y la comunicación con los otros. La literatura se refiere a cada tipo de clasificación con una gran variedad de términos según los momentos y los países; por ejemplo, como sinónimo de "experto", encontramos "académico" (Mercklé 2011), "oficial", "sabio" (Deauvieau et al. 2014). Como sinónimo de "pragmático", encontramos "secular" (Mercklé), "ordinario" (Deauvieau), "indígena" -en el sentido de local, no étnico- (Boutet y Conein 1991). También consideramos en nuestro equipo de investigación el uso de los términos "subjetivo", o "intersubjetivo" cuando nos referimos al resultado de la clasificación en la interacción entre participantes en la investigación. La comparación entre ambas categorizaciones, expertas y pragmáticas, facilitará aproximarnos a la pregunta central de cuáles y cómo son las ideas, conocimientos, distinciones y categorías sociales naturalizadas por las personas acerca de su vida social habitual.

\section{La clase social como categoría de representación de la sociedad}

En el marco de este problema epistemológico, la historia del concepto de clase social ha llevado a la elaboración de uno de los corpus más amplios de las ciencias sociales. El uso de esta noción como categorización y representación de la realidad social ha levantado mucho debate tanto en el campo de las ciencias sociales como de la epistemología de las ciencias sociales. De hecho, la pregunta que surge en todos los esquemas contemporáneos sobre clases sociales es si esas categorías tienen sentido para los individuos en su vida cotidiana y sus esquemas cognitivos (Boutet y Conein 1991). Esto constituye el componente más elemental o primario del problema e implica responder preguntas centrales: ¿Qué creen las personas acerca de los integrantes de la sociedad a la que pertenecen? ¿Qué principios organizan su pensamiento sobre la estructura social? Estas preguntas, situadas a un nivel descriptivo, constituyen nuestro objeto de estudio en este trabajo y son previas a explicar qué determina lo que se cree y cuáles son los efectos de esas creencias para los individuos o para la sociedad (1).

Consolidado por los fisiócratas como término en el momento de la llustración para referirse a la burguesía -el nuevo grupo pujante que desafiaba los estamentos y órdenes fijos del Antiguo Régimen- la clase social como concepto termina sustituyéndose a los órdenes o los estamentos, con el paso a la era industrial. Desde Marx, la relación entre la clase como objeto experto y como objeto -o sujeto social- ha sido uno de los principales focos de discusión, en especial su reflexión acerca de la percepción de parte 
de las personas respecto de la realidad de estos grupos o su conciencia de los mismos. A diferencia de los órdenes, que son espacios sociales dados, en los cuales la pertenencia es adscriptiva e inmóvil, la clase social es un grupo móvil en sus contornos e integrantes, que describe no solamente a las personas que pertenecen a un mismo grupo social por su relación a los medios de producción o por su ocupación y lugar en el mercado laboral (Weber), sino que responde, al igual que la noción de orden, a una visión jerárquica de la sociedad, donde existe interdependencia entre clases sociales. Incluye también una dimensión performativa de cambio social, en especial en la tradición marxista, al invocar en las clases sociales una posibilidad de cambio histórico. Más adelante, el modelo de clases sociales de Weber plantea la existencia de variados clivajes en la conformación de clases sociales, como la propiedad de factores de producción y la situación en el mercado de trabajo y de bienes. Esto ha conducido a enfoques neo-weberianos basados en la desagregación de múltiples dimensiones, como ingresos, educación, que cuando se correlacionan débilmente generan una "inconsistencia de estatus", la que se expresa en una también débil identificación de clase y un carácter más individual de la clase social, en cuanto situación de mercado. En base a estos linajes teóricos, la clase social puede ser definida como "agregados de grupos de un estatus aproximadamente igual en el sistema de estratificación", de acuerdo a Parsons (1951:163), quien enfatiza así en el componente intersubjetivo de la noción de clase social.

Esta reflexión será retomada por Bourdieu en sus trabajos epistemológicos acerca de las nociones de campo y de espacio social, pues considera crucial "la pregunta acerca de las relaciones entre las clasificaciones con pretensión objetiva que produce el experto, parecido en este sentido al zoólogo, y las clasificaciones que los agentes mismos producen sin cesar en la existencia cotidiana [...] o los principios mismos según los cuales estas clasificaciones son producidas" (Bourdieu 1984:5). Desde este punto de vista, cualquier clasificación experta debería reflejar con cierta precisión las representaciones que las personas se hacen del espacio social, en base a su posición en relación con diversas formas de capital, aunque se generen ciertos niveles de distorsión entre ambos ámbitos. Más recientemente, Boltanski y Thévenot desplaza la reflexión desde el conocimiento experto elaborado por los cientistas sociales hacia el conocimiento predominante en las clasificaciones "oficiales", en particular las que produce el aparato estatal, y en las nomenclaturas utilizadas por las empresas (Boltanski y Thévenot 1991). Argumentan que estas nomenclaturas son parte central del sistema de justificación y legitimación del capitalismo actual, lo que denomina "espíritu del capitalismo" (Boltanski y Chiapello 1999). Ese tipo de categorizaciones expertas, como las clasificaciones utilizadas en las políticas públicas y por organismos internacionales, en los estudios académicos y de marketing, son distintivas de la modernidad y ejercen una marcada influencia en la vida social, puesto que es con la modernidad que el nombrar y clasificar se torna una de las funciones centrales del aparato estatal y de la ciencia en especial. Por lo tanto, lo relevante es en qué medida la representación de las propias personas se acerca o aleja de esas categorizaciones expertas, justificando o criticando el orden establecido (Boltanski 2009).

Cabe señalar adicionalmente que existe un hecho cultural que subyace esta discusión: más allá de que los individuos y los colectivos elaboran representaciones taxonómicas de la sociedad, las variables que se usan para ello, como por ejemplo la ocupación, la educación o los ingresos, a su vez "son el objeto de clasificaciones de segundo orden (tratamiento estadísticos, registro administrativo, censos demográficos)" (Boutet y Conein 1991:21), que también circulan entre categorías expertas y categorías pragmáticas. En términos concretos, podemos señalar que la existencia de diversas formas de representación de la sociedad tiene consecuencias directas sobre la realidad. Por ejemplo, en los casos de Gran Bretaña y Francia, donde existen clasificaciones de larga data y en el último caso muy difundida en el conjunto de la sociedad y usada por sus miembros en la vida cotidiana, el debate sobre desigualdades sociales está mucho más nutrido que en países como Alemania, donde no existen tales 
clasificaciones consensuadas y donde se considera que existe una menor legibilidad de "lo social" (Deauvieau et al. 2014). Esto demuestra que "estos esquemas de representaciones de las estructuras sociales varían de manea asombrosa en función de la historia y de la cultura que los conciben. Para el caso alemán, la constatación es que las categorías que maneja la estadística pública no son "sociológicamente pertinentes" (Pfeuffer y Schultheis 2002:17), es decir, no reflejan cognitivamente categorías sociales identificables y ancladas en la realidad cotidiana de las personas. En el caso de Chile, este tipo de reflexión aún no dispone de un corpus importante.

Finalmente, existe desde hace tres décadas un debate candente a nivel internacional respecto de si la clase social sigue siendo la categoría más potente para describir el espacio social, tanto desde las categorías expertas como desde las categorías pragmáticas, es decir, si sigue siendo "uno de los modos de lectura" del mundo social (Mercklé 2011). La polémica se inicia en los años 1950 con los trabajos de Nisbet sobre el fin de las clases sociales en su dilución en la sociedad de masas post guerra, en el caso de Estados Unidos. Es profundizada posteriormente con los trabajos de Bell sobre la sociedad postindustrial, quien señala que el eje central de diferenciación social pasa a ser la calificación, definiendo de ahora en adelante situaciones de status, que se distancian de la noción de clase en su versión marxista. La profecía de la desaparición de las clases como eje clasificatorio se ha repetido hasta los años 1990 en una amplia variedad de estudios. La consolidación en los debates científicos de la potencia del eje sexo/género, diferencias étnicas o generacionales para explicar las principales estructuraciones de lo social, o incluso la aparición de claves de lectura no necesariamente jerárquicas como la conformación en redes, establecen una fuerte competencia con el concepto de clase social, señalando que éste no da cuenta de la totalidad o de una parte suficiente de la realidad social.

Sin embargo, desde la escuela de Frankfurt, hasta la constatación en los años 1980 que las desigualdades están nuevamente en aumento dentro de los países, entre países y en la totalidad del planeta, la reflexión sobre la capacidad de la noción de clase social de dar cuenta de las jerarquías sociales y del espacio social ha sido nuevamente y altamente nutrida, hasta el día de hoy, con debates encendidos al respecto. Recientemente, se llega a considerar que la clase social como concepto no logra capturar a cabalidad el aumento explosivo de las desigualdades en las últimas décadas (Beck 2013), ni por lo tanto ofrecer esquemas y categorizaciones satisfactorios para describir lo social, desafiando de esta forma el "monopolio epistemológico" de los análisis de clase. Tampoco permitiría capturar el hecho que las personas tienen visiones de la sociedad variadas según la posición que ocupan en ella (Boutet), es decir, la clase social no permitiría dar cuenta de la percepción diferencial de la estructura social de los sujetos (Coxon). El debate está abierto, y varios autores consideran que el poder explicativo que alguna vez tuvo el concepto, tanto para las personas comunes y corrientes como para la comprensión experta de las desigualdades, requiere adaptaciones y revisiones.

\section{La medición de las clases sociales y la relación entre objeto y sujeto}

Con el fin de profundizar en la relación entre objeto y sujeto, presentaremos ahora muy brevemente formas de medición de las clases sociales. Al respecto, en la medida en que se refinan las mediciones en ciencias sociales, se complejizan los algoritmos para la construcción de las categorías y se adaptan a contextos nuevos con fines comparativos, así entonces constantemente resurge la pregunta de hasta qué punto estas clasificaciones resuenan en la realidad social y logran capturar las representaciones pragmáticas de las personas. En este contexto, cabe mencionar la clásica reflexión del sociólogo polaco Ossowski (1972) acerca de los modos de clasificación de las clases sociales como paso que antecede la medición. Para ello, sistematiza las metáforas espaciales que se usan para caracterizar a las clases 
sociales. Ossowski, al igual que autores más recientes como Lorenzi-Cioldi y Joye (1988), señala que cualquier medición implica pensar primero la representación de la sociedad: existen por un lado los esquemas de gradación, que corresponden a una representación continua de la sociedad entre los menos dotados y los más dotados, según un ordenamiento unidimensional "simple" (el ingreso por ejemplo o de forma más abstracta el "nivel de vida") o multidimensional (sintético). Por el otro lado, existen esquemas de dependencia, cuyo nombre refleja una relación funcional y jerárquica que se establece entre grupos sociales, con separaciones muy claras y excluyentes entre ellos, conocidas como barreras o límites sociales (Lamont y Fournier 1992, Méndez 2008). Otra característica de este tipo de representación de la sociedad es que una persona pertenece a un grupo y por definición no puede pertenecer a otro; en la mayor parte de los esquemas, se trata de una visión polarizada (como por ejemplo en la representación marxista). Para estos esquemas, la separación entre clases puede corresponder a diferencias de riqueza, de poder, de dominación o de explotación según sea la lógica subyacente de repartición de las ventajas sociales, pero en la sociología post-weberiana tiende a aceptarse que se trata de categorías que involucran diferencias en múltiples dimensiones y no solo en una. En tercer lugar, existen los esquemas funcionales, donde las clases, en vez de ser consideradas como antagónicas, son descritas como complementarias, e interdependientes, en base a la función que desempeñan en la sociedad, como es por ejemplo el caso de los estamentos en la sociedad feudal. En definitiva, podemos desprender de lo anterior que en lo fundamental, las clases sociales constituyen una forma de diferenciación categórica (no gradual), jerarquizada y multidimensional.

Hecha esta primera conceptualización acerca de las formas de representación de las clases sociales, cabe señalar que uno de los problemas que enfrentan las ciencias sociales corresponde al diseño de herramientas de observación que permitan dar cuenta adecuadamente y científicamente de los procesos y fenómenos sociales. La complejización de esos instrumentos ha generado un debate epistemológico desde la segunda mitad del siglo XX, en especial debido a que el desarrollo de la estadística y la capacidad de manejar grandes conjuntos de datos, ha llevado a la creación de una serie de clasificaciones expertas, en especial a partir de los años 1970. Partiendo de la distinción básica en el caso chileno, entre pobres y no-pobres (esquema funcional según Ossowski), que dominó el discurso de instituciones gubernamentales y no-gubernamentales durante la década de los años 1990 y gran parte de la década del 2000 como forma de representación de la sociedad, llegamos a la economía, con sus herramientas de medición de la desigualdad, como por ejemplo los percentiles, deciles o quintiles de ingreso. Como representación de la realidad, el esquema económico de medición consiste en un continuo basado en los ingresos, de carácter unidimensional, lineal y gradual, que corresponde también a una de las representaciones señaladas por Ossowski. Igualmente el marketing, con sus herramientas de medición de la capacidad de consumo (2), ha traspasado las barreras de su ámbito de origen para ser ampliamente usado en ciencias sociales e incluso en el mundo social, donde no es inusual escuchar referencias a esta terminología (en especial al sector $A B C 1$ como encarnación de los sectores más ricos en Chile, aunque se use en menor medida las otras letras para describir a los demás grupos sociales). En este caso, se trata de una herramienta bidimensional, que combina un proxy del ingreso (medido a través de bienes en el hogar, como refrigerador, automóvil, secadora, etc.) y el nivel educacional del principal sostenedor del hogar. Estas formas de describir los grupos que integran la sociedad tienen una resonancia en ella, según el principio de la doble hermenéutica.

En sociología, en cuanto a instrumentos de clasificación y medición, varias herramientas han permitido refinar los aportes de Marx y de Weber, mediante la definición de variables, que son agregadas, para la construcción estadística de clases sociales, es decir la "distribución" de los integrantes de la sociedad en varios grupos sociales. En este caso, la base suele ser la ocupación, variable central, pues se considera 
que dice mucho, no sólo acerca de la posición social de la persona, sino que también de su identidad. De hecho, en cualquier conversación, cuando se conoce a una persona, no se le pregunta cuánto gana ni su nivel de educación, sino qué es lo que hace en la vida. La ocupación permite también dar cuenta del nivel de subordinación o autonomía de las personas en relación con las jerarquías sociales. Por estas razones, estas herramientas suelen separar distintos grupos en la sociedad en función del rango de su ocupación en términos de habilidades y nivel educacional, responsabilidad, supervisión de otros trabajadores, prestigio, estatus, ingresos, etc., con separaciones en general muy claras entre trabajadores manuales y no manuales como clivaje fundamental (Parkin) o quienes ejercen autoridad versus quienes no (Dahrendorf). Estos modelos que han surgido desde los años 1950, han sido refinados a partir de los años 1970 y han dado lugar a nuevas clasificaciones en los años 1990, que buscan por ejemplo reflejar el crecimiento y la diversificación del sector servicios (Esping-Andersen), imponiéndose con mayor fuerza dos esquemas principales: el esquema neo weberiano de Erikson y Goldthorpe y el esquema neomarxista de Eric Olin Wright. Ambos esquemas han sido ampliamente probados, adaptados, criticados y aplicados; permiten comparaciones internacionales de gran alcance y con el paso del tiempo han dado lugar a la generación de nuevas series de datos y una mejor comprensión de la evolución histórica de la estructura de nuestras sociedades (3).

La clasificación predominante de corte sociológico, tanto a nivel internacional como en Chile, ha sido elaborada por el equipo británico compuesto por Erikson, Goldthorpe y Portocarero (EGP, según la sigla que se le asocia), de acuerdo a la cual se crean grupos -clases sociales en este caso- separando entre propietarios, cuentapropistas y empleados, es decir la categoría ocupacional, o siguiendo a Marx, la relación con los medios de producción. Es ampliamente usada a nivel internacional así como en Chile. Luego de esta primera distinción, se revisa la situación de quienes están en posición de supervisores para establecer la responsabilidad que tienen en la jerarquía social (lógica de autoridad) y finalmente se separan los casos en base al tamaño del establecimiento en el cual la persona trabaja (lógica de supervisión). Se obtiene una clasificación de siete clases sociales: 1) Clase de servicios: profesionales, administradores y gerentes, técnicos de nivel superior, supervisores de trabajadores no manuales; 2) Trabajadores no manuales de rutina en administración y comercio, personal de ventas, otros trabajadores de servicios (de nivel superior e inferior); 3) Pequeña burguesía e independientes: pequeños propietarios, artesanos, etc. con y sin empleados; 4) Agricultores: propietarios agrícolas y arrendatarios, así como otros trabajadores en cuenta propia en la producción primaria; 5) Trabajadores manuales calificados: técnicos de rango bajo, supervisores de trabajadores manuales, trabajadores manuales calificados en general; 6) Trabajadores manuales semi-calificados y no calificados (no agrícolas); 7) Trabajadores agrícolas y otros en la producción primaria (Erikson et. al).

En definitiva, la escala de Erikson, Goldthorpe y Portocarero (EGP) aplica un criterio principalmente ocupacional pero también otros como la relación con los medios de producción, distingue varios estratos de clases medias y establece claramente una jerarquía, aunque no unidimensional. Nuestra interrogante en este caso, sintetizada en la escala EGP, es resumida por Grusky de esta forma: "La pregunta que se plantea necesariamente para todos estos esquemas contemporáneos es si sus categorías constituyentes son entidades puramente nominales o si tienen realmente un significado para los individuos concernidos" (Grusky 2001:7). En efecto, no todas las clasificaciones tienen una resonancia inmediata en la realidad social ni tienen un igual impacto socio-cultural que conduzca a que las personas en su vida cotidiana usen los nombres de estas clasificaciones para referirse a los grupos que componen el espacio social (4). Algunas formas de representación experta tienen más relación que otras con las formas de representación común de la realidad social, que es lo que pasaremos ahora a analizar. 


\section{¿Cómo superar la distancia entre categoría pragmática y experta? Una propuesta metodológica}

Se han perfeccionado en las últimas décadas los protocolos y las técnicas de investigación, en especial gracias al diálogo entre ciencias sociales y a un trabajo cada vez más reflexivo en metodología, apuntando a un intercambio fructífero con la epistemología. Uno de los espacios de investigación que, sin embargo, requiere mayores esfuerzos desde la sociología, tiene que ver con la observación de las decisiones que toman las personas, en especial las decisiones de tipo cognitivo que permiten organizar mentalmente el mundo en el cual vivimos, sea en un nivel altamente reflexivo o con un menor nivel de reflexividad. En efecto, ¿cómo dar cuenta de la formación de percepciones respecto de las diferencias sociales, tal como ocurren en la mente de las personas o en situaciones de interacción social cotidianas con sus múltiples variantes? ¿Cómo reproducir, por ejemplo, la seguidilla de pensamientos que lleva a una persona a clasificar a otra persona en el mapa que ha elaborado del espacio social? La mera suma de opiniones individuales a través de encuestas poco puede ayudar al respecto. Tampoco las entrevistas individuales, por muy profundizadas que sean, pueden dar cuenta de cómo las personas piensan la sociedad, cómo la perciben y se mueven en ella en el día a día, comunicándose con otros. ¿De qué manera entonces podemos acercarnos al surgimiento de clasificaciones sociales en la mente de los individuos y en su interacción con otros? Proponemos aquí una metodología que retoma preocupaciones etnográficas -aunque no las metodologías de la etnografía-, en el sentido que permite observar cuando las personas describen lo social, mientras lo están haciendo.

Llevamos a cabo un estudio inspirado en un enfoque pragmático, que pone el énfasis en la pluralidad de los modos de acción de los actores sociales, así como en metodologías de experimentación usadas en economía y metodologías interactivas cercanas a una psicología social. En este contexto, realizamos un ejercicio de simulación que permite estudiar clasificaciones compartidas entre las personas, a través de un juego consistente en categorizar, es decir combinar o emparejar elementos semejantes. Nos inspiramos libremente en un juego de este tipo aplicado por Boltanski y Thévenot, buscando "volver a cuestionar la posibilidad y el interés de una clasificación experta universalmente válida" (Deauvieau et al. 2014). Se trata de una metodología innovadora en las ciencias sociales, sin precedentes en la investigación social en Chile o en América Latina (5). Consiste en un juego de naipes desarrollado en grupos focales, para observar en situaciones interactivas de qué modo los individuos clasifican a las personas de la sociedad y cómo describen el espacio social (6), sin que el equipo de investigadores defina a priori qué imagen de la sociedad debería primar, sino que se deja a los participantes -que denominaremos también "jugadores" debido a la naturaleza del ejercicio- elegir sus propias lógicas clasificatorias. La metodología utilizada busca analizar procesos semi-reflexivos que ocurren en situaciones interactivas, las interpretaciones y las lógicas clasificatorias que usan diversos actores y grupos sociales para separar a las personas en los distintos grupos sociales, los argumentos que justifican las clasificaciones, así como las fronteras simbólicas y materiales que separan a las categorías sociales. Cabe señalar que si bien esta aproximación permite lo anterior, nuestro análisis se focaliza principalmente en los mecanismos de diverso grado de reflexividad mediante los cuales las personas realizan las operaciones relativas a nombrar y clasificar, para lo que contamos con el discurso de los jugadores y algunas actitudes como silencios y bromas. El material obtenido también permite un análisis diacrónico, enfocado a distinguir los momentos en que los participantes pasan de un modo altamente reflexivo a uno de menor reflexividad o a la inversa.

El juego está basado en naipes que contienen fotografías y datos de personas reales, que jugadores de distinta procedencia social y sexo deben clasificar según las características que consideren más relevantes. La dimensión lúdica del ejercicio permite mantener a las personas interesadas y 
concentradas por una duración de entre dos y tres horas. A través de esta metodología, entonces, se procura entender los elementos subjetivos que nutren o dan sentido a la existencia las jerarquías sociales en Chile a través de las clasificaciones intuitivas y en variados niveles de reflexividad, que elaboran las personas. Los naipes entregados a los jugadores buscan representar múltiples características de individuos en la sociedad, por lo que cada tarjeta contiene información como ocupación, nivel de educación, tramo de ingreso mensual, comuna de residencia, edad, sexo, religión, origen étnico y otras características, incluyendo una fotografía de la persona. Así, la información presentada en los naipes ofrece numerosas opciones de categorización. Los participantes en el juego fueron personas corrientes que no se conocían entre sí, seleccionadas y organizadas en seis grupos conformados de acuerdo a su pertenencia a una clase social específica (7). En la composición de cada grupo, se aseguró la presencia tanto de hombres como mujeres, así como de personas de distintos rangos etarios.

Se solicitó a los jugadores agrupar los naipes libremente, de acuerdo a sus propios criterios, pues no se dieron consignas específicas, para evitar efectos inducidos. La categorización hecha por los jugadores no era predecible ni obvia, porque la libertad en la elección de estos criterios se vio reforzada por la representatividad de los naipes con respecto de una amplia diversidad de personas en la sociedad. Una vez que los jugadores terminaron de agrupar los naipes en distintas pilas, se les solicitó poner un nombre de su elección a cada agrupación, elegir dentro de cada montón el naipe que les pareciera más representativo de ese conjunto y finalmente ordenar los montones para dar cuenta de sus relaciones mutuas en el espacio social en términos de jerarquía y distancias sociales.

\section{Nombrando los grupos sociales: resultados y reflexiones}

Para el análisis de los resultados del estudio, interesa saber, retomando la reflexión de Ossowski (1972), qué características tiene la descripción interactiva y pragmática de las clases sociales realizadas por los jugadores, en especial si se corresponden con las nociones de dicotomía o de gradación y jerarquía, de oposición entre quienes dirigen y quienes obedecen, entre ricos y quienes no lo son, etc. Es decir, cuáles son los principios que ordenan dicha jerarquía: el poder político, el poder económico, expresado en posesión de los medios de producción, dominación o explotación, u otras lógicas. ¿Qué categorías eligieron entonces los grupos de participantes para agrupar a las personas de la sociedad representadas en los naipes? ¿Existe relación entre estas clasificaciones pragmáticas y las clasificaciones expertas presentadas anteriormente? En la tabla siguiente, indicamos en la columna a la izquierda el nivel socioeconómico de los participantes en el juego, para cada grupo de jugadores y en la columna a la derecha los nombres que los jugadores dieron a los conjuntos de naipes agrupados para describir la sociedad chilena. 


\section{Clasificaciones elaboradas por los integrantes de los grupos}

\begin{tabular}{|c|c|}
\hline $\begin{array}{l}\text { Grupos de participantes (jugadores) según } \\
\text { clase social (EGP) }\end{array}$ & $\begin{array}{l}\text { Nombres de las categorías (agrupaciones de } \\
\text { naipes) elaboradas por los participantes } \\
\text { correspondientes }\end{array}$ \\
\hline $\begin{array}{l}\text { Servicios Alta } \\
\text { Ejemplo: Profesional de nivel alto }\end{array}$ & 1) Oro 2) Plata 3) Cobre 4) Plomo \\
\hline $\begin{array}{l}\text { Servicios Baja } \\
\text { Ejemplo: Profesional de rango medio o bajo }\end{array}$ & $\begin{array}{l}\text { 1) Profesionales exitosos 2) Emprendedores 3) } \\
\text { Oficios varios }\end{array}$ \\
\hline $\begin{array}{l}\text { Rutinas No-manuales Alta } \\
\text { Ejemplo: Empleado administrativo }\end{array}$ & $\begin{array}{l}\text { 1) Ganadores 2) Diversidad 3) Superación 4) } \\
\text { Conformistas 5) Estancados 6) Jubilados 7) } \\
\text { Dueñas de casa }\end{array}$ \\
\hline $\begin{array}{l}\text { Rutinas No-manuales Baja } \\
\text { Ejemplo: Secretaria }\end{array}$ & 1) Universitarios 2) Medio 3) Bajo \\
\hline $\begin{array}{l}\text { Independientes y Pequeños Empresarios } \\
\text { Ejemplo: Comerciante pequeño }\end{array}$ & $\begin{array}{l}\text { 1) Exitosos y autosuficientes 2) Profesionales } \\
\text { jóvenes inestables } 3 \text { ) Clase media esforzada 4) } \\
\text { Conformistas y resignados 5) Reinas } \\
\text { dependientes }\end{array}$ \\
\hline $\begin{array}{l}\text { Trabajadores Manuales No-calificados } \\
\text { Ejemplo: Trabajador de la construcción }\end{array}$ & $\begin{array}{l}\text { 1) Mejor estrato social 2) Superados 3) } \\
\text { Esforzados }\end{array}$ \\
\hline
\end{tabular}

Nota: una completa explicación de esta tabla y otros aspectos metodológicos se pueden encontrar en otros artículos o en el sitio web www.desigualdades.cl, pues nuestro objetivo aquí es abordar los elementos epistemológicos y no empíricos de la investigación.

En términos generales, las clasificaciones elaboradas por los seis grupos de jugadores expresan una jerarquía no dicotómica y están ordenadas entre posiciones superiores e inferiores. Ninguno de los grupos cuestionó la existencia de una jerarquía social y los jugadores construyeron diferencias entre categorías con relativa facilidad, lo que da cuenta de la relevancia de las tareas de categorización en la vida social como parte de la dialéctica de la identificación (Jenkins 2000). Los diferentes grupos establecieron entre tres y siete montones de naipes que representan los principales grupos en el espacio social. Aunque las clasificaciones resultantes son todas distintas, como era de esperar debido a que las representaciones de la sociedad tienden a depender de la posición de quien describe la sociedad, en el proceso de agrupación de los naipes, los participantes aplicaron en general criterios comparativos y jerárquicos, como "ganan menos que" y "tienen menos educación que", es decir una lógica de cuantificación que se asemeja a la de las categorías expertas, aunque no con el mismo nivel de desarrollo. En la mayor parte de los casos, las clasificaciones fueron elaboradas con rapidez y muchas veces con acuerdos rápidos, lo que refleja los hábitos no reflexivos y rutinarios o habituales de categorización en la vida social. Las lógicas más reflexivas de categorización se manifestaron con mayor fuerza al inicio del juego o en los momentos de conflicto entre los participantes. 
En segundo lugar, los participantes recurrieron en términos de su descripción del espacio social sobre todo a una agregación y ordenamiento propio de múltiples dimensiones, en especial de variables relacionadas primero que nada con educación y luego una mezcla de ingresos y ocupación. El peso de la educación es particularmente notorio, en especial la separación que se establece entre profesionales y no profesionales, sin lugar a dudas, una de las dicotomías más fuertes. Esto refleja en parte el poder que ha adquirido la educación en el lenguaje y la realidad social chilena y latinoamericana en los últimos treinta años, como visión de una posible y deseada movilidad social ascendente y como diferenciador en términos de oportunidades laborales y económicas. También da cuenta del desplazamiento de la centralidad del trabajo en la comprensión de la estructuración social en las últimas décadas, en comparación con el período transcurrido hasta los años 1960. Incluso, el campo educacional y cultural adquiere una singular relevancia en este caso, frente al campo meramente económico, que ha dominado los estudios de estratificación, en especial en economía. La posición que ocupa la educación en las clasificaciones pragmáticas en Chile es un hallazgo relevante en cuanto principio ordenador de la jerarquía social, en comparación con Europa y Estados Unidos, pero también en cuanto al orden institucional y el lugar donde los actores sociales ponen el énfasis en su comprensión de la sociedad. Subyace una pregunta política relevante sobre qué tipo de recursos son los que se valoran o penalizan en la sociedad. En menor medida que el nivel educacional, se evidencia en las clasificaciones otras variables clasificatorias, principalmente la clase social según ocupación y los ingresos, por lo que existe a su vez una jerarquía entre principios clasificatorios. En definitiva, las personas usan clasificaciones abstractas parecidas entre sí y que recurren a una combinación de varias dimensiones de análisis, es decir a esquemas sintéticos.

En lo que se refiere a la relación entre categorías expertas y categorías pragmáticas, llama la atención que solo en una de las clasificaciones se usa la palabra clase ("clase media esforzada"). Pareciera ser por lo tanto que este concepto no constituye la forma preferente en que las personas denominan los grandes grupos que se conforman en la sociedad, aunque el término sí aparece de forma recurrente en las conversaciones entre los participantes. Para profundizar en este aspecto, podemos señalar que otras dimensiones relevantes en lo social y en las clasificaciones de clases, no aparecen en esta clasificación, como por ejemplo la noción de poder, que sí está presente en esquemas expertos, mediante nociones como subordinación, dominación, autonomía o supervisión. En cuanto a variables adscriptivas, como la edad o la generación -salvo en la mención "profesionales jóvenes inestables" de uno de los grupos-, el origen étnico o el lugar de residencia, aunque se trataba de información disponible para los jugadores y que representan ejes importantes de estructuración de la sociedad chilena y de muchas sociedades, no son relevantes en el resultado de la clasificación. Se ve que al igual que en las clasificaciones expertas, se privilegian ciertas dimensiones que son más accesibles cognitivamente, permiten más fácilmente organizar clasificaciones o sencillamente se encuentran más discutidas en la vida social y reconocidas por los actores, de tal manera que se hace posible usarlas con mayor facilidad. Respecto del sexo, otra variable adscriptiva, muy relevante en la estructuración social en Chile y en especial en lo laboral, sólo se usa para diferenciar a las mujeres sin ocupación formal y que se dedican a las labores de la casa. En dos grupos, se identifica una categoría de dueñas de casa ("dueñas de casa" y "reinas dependientes"), lo que refleja la gran cantidad de mujeres en esta condición en Chile. Tampoco se evidencia en las clasificaciones la importancia del origen étnico, aunque era un elemento que se podía extraer de los naipes y que es de extrema relevancia para la legibilidad de lo social y de sus jerarquías en América Latina, aunque rara vez sea usado en esquemas expertos. Cabe señalar en todo caso que esos elementos sí aparecen en las conversaciones entre los jugadores en momentos específicos, cuando abordan la 
diversidad dentro de las categorías o en relación con naipes específicos, aunque no sean las dimensiones estructurantes de las clasificaciones.

Los resultados muestran que las personas en los naipes son clasificadas por los jugadores de acuerdo a un pequeño número de grandes categorías sintéticas que reflejan un ordenamiento de la sociedad. Esta categorización se aproxima a la lógica de una clasificación según clases sociales al estilo de la escala de Erikson, Goldthorpe y Portocarero (EGP), pues las categorías tienen un carácter multidimensional o sintético, con énfasis en uno u otro recurso, en lugar de un recurso único como los ingresos, la ocupación o la educación. Adicionalmente, todos los grupos distinguen más de una clase media y cada grupo las categoriza de un modo particular, conformando así múltiples clases medias, de modo también similar a la corriente predominante en sociología (8). Finalmente, establecen una posición jerárquica entre diversos estratos de clase media y de éstos con la categoría inferior, integrada principalmente por trabajadores manuales, siendo la jerarquización un rasgo básico de una estructura de clases sociales. A pesar entonces de las diferencias entre categorías expertas sobre clases sociales y categorías pragmáticas, existen similitudes en cuanto a que las categorizaciones elaboradas por los jugadores se aproximan a una clasificación sintética de clases sociales, en que las personas distinguen mayoritariamente más de una categoría de clases medias y se establece una jerarquía entre las categorías. La noción de clase media, bajo diversas denominaciones, se encuentra bien establecida en el discurso y el diálogo corriente entre las personas, por lo que debe ser tomada en consideración. Ciertamente, la noción de clase media ha sido construida no solamente en forma autónoma por los chilenos y chilenas, sino que es producto también de los medios de comunicación, de los estudios y estrategias de marketing y de la herencia de un discurso histórico sobre grandes categorías sociales, pero la construcción subjetiva no se enmarca exclusivamente en esas influencias ni sigue una pauta única. En todo caso, permanece abierta la pregunta de si la distinción subjetiva entre múltiples clases medias, al ocurrir bajo formas heterogéneas de nombrarlas, implica subjetivamente una misma representación simbólica.

Otro elemento merece también ser señalado: la dimensión normativa de las clasificaciones. Las categorías elaboradas por las personas -los participantes en los grupos, en nuestro ejercicio-, expresan en un plano cognitivo compartido la agrupación y ordenación de múltiples dimensiones para clasificar a los integrantes de la sociedad. Es decir, la categorización subjetiva consiste en una combinación de un conjunto de ordenamientos en un único orden general: en su clasificación, las personas utilizan una nomenclatura que constituye una representación aproximada de su percepción de las diferencias sociales, expresando a través de los nombres de cada categoría una conexión según semejanzas, más que un orden nítido y preciso. No obstante, más allá de la actividad meramente clasificatoria, en el diálogo de los grupos acerca de las clasificaciones, hubo un marcado componente normativo (Durhkeim, Parsons), que no está presente en las clasificaciones expertas y que califica positivamente o negativamente las posiciones de las personas en la sociedad. En efecto, las clasificaciones expertas actuales en general son más bien cognitivas y descriptivas: no incluyen o no explicitan juicios valóricos de los autores acerca del orden social, de su justicia o injusticia. En cambio, como se puede apreciar en las clasificaciones pragmáticas, la gente común y corriente incluye en sus definiciones juicios de este orden. Durkheim y Mauss ya señalaban la importancia de la significación moral de las clasificaciones sociales, más allá de su mera dimensión realista, enfatizando su dimensión favorable o desfavorable, deseable o no deseable. Como se puede observar en el juego, las personas moralizan, siguiendo los patrones siguientes: "los de allá son exitosos, los de acá son esforzados", usando la vara de lo que consideran que debería ser el comportamiento y la posición de los demás en la sociedad, así como cuál debería ser la conformación del espacio social. Como se puede apreciar en las nomenclaturas utilizadas por los 
jugadores, se incluyen fuertes elementos morales o que refieren a valores sociales y al respeto social que le correspondería a cada personaje representado en los naipes, según lo definen los jugadores. Constituyen un elemento central para las clasificaciones elaboradas por los jugadores ("superación", "emprendedores" en su versión positiva; "conformistas", "estancados", en su versión negativa o estigmatizadora), aunque no sean fáciles de aislar de las lógicas de clasificación y jerarquización señaladas anteriormente, como por ejemplo el nivel educacional o la posición ocupacional. La noción de esfuerzo, es decir el compromiso y la capacidad de trabajo y/o el empeño por educarse, que despliegan supuestamente las personas, constituye un referente ético que como se puede apreciar, estructura varias clasificaciones. En el mismo plano, opera la valoración positiva del éxito social ("profesionales exitosos", "exitosos y autosuficientes"), del acceso a oportunidades ("diversidad"), en una sociedad donde los valores neoliberales han plasmado profundamente. Cabe señalar por lo tanto que en la comprensión de la sociedad, la categorización de sus integrantes en grandes grupos refleja a la vez un ejercicio de descripción y de evaluación normativa, de modo que ambas evaluaciones son difícilmente separables en la percepción subjetiva y cotidiana de la realidad social.

Cabe señalar finalmente que estos hallazgos son transversales para los seis grupos, lo que muestra una cierta homogeneidad de los criterios de clasificación en la sociedad chilena en su conjunto o por lo menos tal como está representada a través de las seis clases sociales que participaron en el juego. Muestra que existe claramente una "jerarquía de los principios de jerarquización" en la descripción de la realidad social (Bourdieu 1984:10).

\section{Conclusión: el arte de la clasificación y la correspondencia entre categorías expertas y categorías pragmáticas}

En este trabajo, buscamos reflexionar desde la epistemología de las ciencias sociales acerca de las representaciones y categorizaciones que se usan para describir el espacio social. Partiendo de la idea que la categorización es indispensable para la comprensión del mundo social, nos centramos en las relaciones entre categorías expertas y pragmáticas, en especial enfocando si son parecidas, se reflejan mutuamente o si más bien responden a lógicas clasificatorias distintas. En este ejercicio, podemos comprobar que el "arte" de la clasificación tiende a reflejar imágenes compartidas de la sociedad entre expertos y sujetos, en especial a través del uso de la educación y en menor medida de la clase social mediante ocupación e ingresos, habitualmente usadas en las categorizaciones expertas. Las formas específicas de nombrar a cada una de las categorías sociales se alejan de la nomenclatura experta tal como la reseñamos respecto de la escala según ocupaciones de Erikson, Goldthorpe y Portocarero (EGP), una de las clasificaciones más difundidas, pero también de la distinción pobres-no pobres, de un continuo basado en los ingresos y de las categorías aplicadas en marketing en Chile. Cabe subrayar que la ocupación está presente en las clasificaciones subjetivas, de modo similar a categorías expertas acerca de clases sociales. Sin embargo, en el caso chileno la educación es tanto o más importante que el criterio ocupacional. Las diferencias o distinciones significativas que perciben y reconocen los jugadores en su universo social no son todas las dimensiones "objetivas" puestas a su disposición por las instituciones académicas, económicas y oficiales, sino que predominantemente las relacionadas con la educación (objetiva) y el éxito o esfuerzo (a la vez objetivo, subjetivo y normativo), estableciendo así un orden y una jerarquía simbólica, que a la vez representan y reflejan las estructuras y procesos de la sociedad.

Si bien no existe una total adecuación entre clasificaciones expertas y clasificaciones pragmáticas, comparten una serie de lógicas de categorización, donde se observa una misma semántica acerca del mundo social. Adicionalmente, la dimensión normativa, que no está presente de modo explícito en las 
categorizaciones expertas, se hace claramente notar como eje de clasificación en las categorías pragmáticas. Las clasificaciones elaboradas por los participantes tienen un carácter sintético en el sentido de que incluyen múltiples dimensiones descriptivas -como en las categorizaciones científicas-, a lo que se agrega la inclusión de una valoración normativa ajena a las clasificaciones expertas. Para los participantes, lo multidimensional tiene el carácter de una aproximación, pues es más incierto y de contornos más ambiguos que una definición experta, por lo que el juicio moral atenúa esa incertidumbre a través de una eticidad legítima.

El debate sobre qué son las clases sociales y la discusión sobre su relevancia y validez en cuanto categorías de descripción y categorización de la sociedad actual, tiene múltiples respuestas. Pero la amplitud de lo discutido se acota al pasar desde la teoría al mundo de lo subjetivo, donde hemos observado que el sentido común se acerca más de lo que se cree a la interpretación de la corriente predominante en las ciencias sociales. En esta ocasión, pudimos observar cómo los procesos de categorización no reflejan una visión dicotómica ni tampoco gradualista acerca de la sociedad. Retomando a Giddens y Bourdieu, podemos señalar que la percepción del mundo social implica un acto de construcción a la vez mental y social, que opera en la práctica en base a representaciones sociales disponibles y moldeables en las interacciones sociales. Reflejan también la incorporación de las estructuras objetivas de la sociedad. Las operaciones mentales relacionadas con nombrar y clasificar, como señalan Durkheim y Mauss, significan recurrir a operaciones mentales complejas y en base a analogías muy especiales, que implican relaciones de jerarquía, semejanza o diferencia. La elaboración de categorías que describen el espacio social resulta del desafío de construir colectivamente clasificaciones sociales que sirvan a la vez para propósitos científicos y políticos, pero también remitan a la comprensión del mundo social de parte de los actores y a la posibilidad de volver inteligibles y lógicas las relaciones entre sujetos. Como hemos mostrado, la diferencia más relevante entre las categorizaciones expertas y las de las personas en su vida cotidiana, radica en la valoración moral que estas últimas incorporan y no tanto en la forma de comprender la sociedad, lo cual confiere relevancia a las clases sociales como forma de nombrar y clasificar, vinculante entre analistas y actores.

\section{Agradecimientos}

Este artículo se enmarca en los proyectos FONDECYT/1130276, Enlace VID-CEPIA y FONDAP/15130009/COES. Agradecemos el financiamiento de Conicyt para la realización de esta investigación y de la Universidad de Chile para el proyecto de continuidad. También agradecemos los aportes y comentarios a este documento de los evaluadores anónimos y del editor, así como de Virginia Guzmán, María Luisa Méndez y Carolina Pinto. También agradecemos los intercambios con Cécile Brousse y Etienne Penissat acerca de estas problemáticas.

\section{Notas}

(1) En este sentido, no nos referiremos a los debates sobre relación entre clase social, clase en sí y clase para sí o sobre la conciencia de clase, que también podemos llamar clase "auto percibida". Tampoco nos referimos a otro debate que tiene que ver con el valor social o prestigio asociado a determinadas posiciones u ocupaciones en la sociedad. Tampoco nos referimos al campo de la clasificación desde el punto de vista de la lingüística, que cuenta con un amplio corpus de investigación.

(2) Con los grupos $A B C 1$, que corresponde a los sectores altos, $C 2$, que corresponde a la clase "mediamedia", C3, que corresponde a la clase media baja, D, que corresponde a los sectores populares y E, que corresponde a los pobres. 
(3) Estas clasificaciones usan encuestas propias o encuestas producidos por otros. En general, tal como en los esquemas anteriores, usan la variable ocupación definida y clasificada por la Organización Internacional del Trabajo.

(4) A la inversa, un ejemplo clásico es el esquema usado en Francia por el aparato estadístico -las "categorías socio-profesionales"- desde el final de la segunda guerra mundial hasta los años 1990, que documentadamente presentó un grado de adecuación muy alto con las categorías usadas por las personas para describir el espacio social, mientras que el esquema EGP ha sido criticado por ser menos legible de parte de los sujetos (Brousse et al. 2010, Deauvieau et al. 2014).

(5) Se aplicó en los primeros meses del año 2012. El ejercicio de simulación se detalla en un documento de trabajo de Mac-Clure et. al., disponible en: http://www.desigualdades.cl/wpcontent/uploads/2011/11/Mac-Clure-et-al-Metodolog\%C3\%ADa-juego-de-clasificaciones-mayo2012.pdf.

(6) Una versión parecida de este juego, además de la de Boltanski y Thévenot (1983) corresponde a la encuesta "Describir la sociedad" aplicada en Francia (Pénissat y Jayet 2009) y una aplicación reciente limitada en varios países de la Unión Europea, incluidos Alemania, Bélgica, España y Polonia.

(7) La decisión de separar por grupos de clase se debe a que estudios anteriores (Coxon y Jones 1979) demostraron que las concepciones del mundo social, en especial de su estratificación, dependen de manera muy fuerte de quienes evalúan dicho mundo. Los seis grupos organizados abarcaron un total de 36 jugadores, residentes en la Región Metropolitana de Santiago.

(8) En estas clasificaciones, no está representada la élite económica o "clase patrimonial" que es en realidad la clase superior. Los jugadores que identificaron tres grupos están describiendo una clase media-alta, más que una clase alta como tal.

\section{Bibliografía}

Beck, U. 2013. Why 'class' is too soft a category to capture the explosiveness of social inequality at the beginning of the twenty-first century. The British Journal of Sociology 64(1): 63-74. doi: 10.1111/14684446.12005

Boltanski, L. 2009. De la critique. Précis de sociologie de l'émancipation. Paris: Editions Gallimard.

Boltanski, L. y Chiapello, È. 1999. Le nouvel esprit du capitalisme. Paris: Gallimard.

Boltanski, L. y Thévenot, L. 1983. Finding one's way in social space: a study based on games. Social Science Information 22(4-5): 631-680. doi: 10.1177/053901883022004003

Boltanski, L. y Thévenot, L. 1991. De la justification. Paris: Gallimard.

Bourdieu, P. 1984. Espace social et genèse des "classes". Actes de la recherche en sciences sociales 52-53: 3-14. http://www.persee.fr/web/revues/home/prescript/article/arss_0335-

5322_1984_num_52_1_3327

Boutet, J. y Conein, B. 1991. Catégorisations dans le langage. Paris: Université Paris 7. http://www.langage.travail.crg.polytechnique.fr/cahiers/Cahier_1.pdf 
Brousse, C. et.al. 2010. Assessment of the European socio-economic classification prototype (EseC): lessons from the French experience. Paris: Institut National de la Statistique et des Études Économiques (INSEE). http://www.insee.fr/fr/publications-et-services/docs_doc_travail/docf1006.pdf

Coxon, A. y Jones, C. 1979. Image and predication: the use of subjective occupational hierarchies. Quality and Quantity 13(2): 121-140. doi: 10.1007/BF00139633

Deauvieau, J, Penissat, E, Brousse, C y Jayet, C. 2014. Les catégorisations ordinaires de l'espace social français. Une analyse à partir d'un jeu de cartes. Revue Française de Sociologie 55(3): 411-457.

Durkheim, E. y Mauss, E. 1903. De quelques formes de classification - contribution à l'étude des représentations collectives. Année Sociologique 6: 1-72.

Giddens, A. 1993. New rules of sociological method. Stanford: Stanford University Press.

Grusky, D. 2001. Social stratification: class, race and gender in sociological perspective. Boulder: Westview Press.

Hacking, I. 1999. The social construction of what? Cambridge: Harvard University Press.

Jenkins, R. 2000. Categorization: identity, social process and epistemology. Current Sociology 48(3): 7-25. doi: $10.1177 / 0011392100048003003$

Joignant, A. y Guell, P. 2009. El arte de clasificar a los chilenos. Santiago: Ediciones UDP.

Lamont, M. y Fournier, M. 1992. Cultivating differences. Symbolic boundaries and the making of inequality. Chicago: University of Chicago Press.

Lorenzi-Cioldi, F. y Joye, D. 1988. Représentations sociales de catégories socioprofessionnelles: aspects méthodologiques. Bulletin de psychologie 41(384): 377-390.

Méndez, M.L. 2008. Middle class identities in a neoliberal age: tensions between contested authenticities. The Sociological Review 56(2): 220-237. doi: 10.1111/j.1467-954X.2008.00785.x

Merklé, P. 2011. Qui a fait disparaître les classes sociales? Disponible en: http://pierremerckle.fr/2011/10/qui-a-fait-disparaitre-les-classessociales/?PHPSESSID=324d0edf223eead1065f245931ba7ba8

Mervis, C.B. y Rosch, E. 1981. Categorization of natural objects. Annual Review of Psychology 32: 89-115. doi: 10.1146/annurev.ps.32.020181.000513

Moscovici, S. 1979. El psicoanálisis, su imagen y su público. Buenos Aires: Huemul.

Ossowski, S. 1972. Class structure in the social consciousness. New York: The Free Press.

Parsons, T. 1951. The social system. London: Routledge \& Kegan Paul Ltd.

Penissat, E. y Jayet, C. 2009. "Décrire la société". Une enquête pour comprendre les logiques de classification et de perception de l'espace social. Rapport rédigé pour l'INSEE. Paris: Maurice Halbwachs. 
Pfeuffer, A. y Schultheis, F. 2002. Quelques particularités allemandes dans la représentation statistique du monde social. Sociétés contemporaines 1 (45-46): 17-42. doi: 10.3917/soco.045.0017

Salès-Wuillemin, E. 2007. Catégorisation et représentations sociales. En: M. Bromberg y A. Trognon (Eds.) Cours de psychologie sociale. Paris: Presses Universitaires de France, pp. 7-32. https://halshs.archives-ouvertes.fr/halshs-00903240

Swidler, A. y Arditi, J. 1994. The new sociology of knowledge. Annual Review of Sociology 20: 305-329. doi: 10.1146/annurev.so.20.080194.001513

Recibido el 13 Jul 2014

Aceptado el 25 Sep 2014 